\title{
Consensus Report From the 6th International Forum for Liver MRI Using Gadoxetic Acid
}

\author{
Claude B. Sirlin, MD, ${ }^{1 *}$ Hero K. Hussain, MD, ${ }^{2}$ Eduard Jonas, MD, PhD, ${ }^{3}$ \\ Masayuki Kanematsu, MD, PhD, ${ }^{4}$ Jeong Min Lee, MD, ${ }^{5}$ Elmar M. Merkle, MD, ${ }^{6}$ \\ Markus Peck-Radosavljevic, MD, ${ }^{7}$ Scott B. Reeder, MD, PhD, ${ }^{8}$ \\ Jens Ricke, MD, ${ }^{9}$ and Michiie Sakamoto, MD, $\mathrm{PhD}^{10}$
}

\begin{abstract}
As the utility of liver-specific magnetic resonance imaging (MRI) increases, it is pertinent to optimize and expand protocols to improve accuracy and foster evolution of techniques; in turn, positive impacts should be seen in patient management. This article reports on the latest expert thinking and current evidence in the field of liverspecific MRI, as discussed at the $6^{\text {th }}$ International Forum for Liver MRI, which was held in Vancouver, Canada in September 2012. Topics discussed at this forum described the use of gadoxetic acid-enhanced MRI for the assessment of liver function at the segmental level; to increase accuracy in the diagnosis of liver metastases; to overcome current challenges in patients with cirrhosis, including management of arterial hypo-/isovascular, hepatobiliary phase hypointense nodules; and the data which would be required in order to recommend the use of this modality in hepatocellular carcinoma management guidelines. Growing evidence suggests that gadoxetic acid-enhanced MRI can help to improve the management of patients with a number of different liver disorders; however, more data are needed in some areas, and there may be a case for developing an interpretation guideline
\end{abstract}

${ }^{1}$ Liver Imaging Group, Department of Radiology, University of California, San Diego, California, USA.

${ }^{2}$ Department of Radiology, University of Michigan, Ann Arbor, Michigan, USA.

${ }^{3}$ Department of Upper Abdominal Surgery, Karolinska University Hospital, Stockholm, Sweden.

${ }^{4}$ Department of Radiology, Gifu University Hospital, Gifu, Japan.

${ }^{5}$ Department of Radiology, Seoul National University Hospital, Seoul, South Korea.

${ }^{6}$ Department of Radiology, University Hospital Basel, Basel, Switzerland.

${ }^{7}$ Department of Gastroenterology and Hepatology, General Hospital and Medical University of Vienna, Vienna, Austria.

${ }^{8}$ Departments of Radiology, Medical Physics, Biomedical Engineering and Medicine, University of Wisconsin, Madison, Wisconsin, USA.

${ }^{9}$ Department of Radiology and Nuclear Medicine, Universität Magdeburg, Magdeburg, Germany.

${ }^{10}$ Department of Pathology, Keio University School of Medicine, Tokyo, Japan.

Contract grant sponsor: Bayer HealthCare.

*Address reprint requests to: C.B.S., University of California, San

Diego, 408 Dickinson Street, San Diego, CA 92103-8226.

E-mail: csirlin@ucsd.edu

Received June 5, 2013; Accepted August 21, 2013

DOI 10.1002/jmri.24419

View this article online at wileyonlinelibrary.com. for gadoxetic acid-enhanced MRI findings to aid standardization.

Key Words: International forum for liver MRI; consensus report; gadoxetic acid; gadoxetic disodium; liver function; liver metastases; hepatocellular carcinoma management guidelines; cirrhosis

\section{J. Magn. Reson. Imaging 2014;40:516-529.}

(c) 2013 Wiley Periodicals, Inc.

THE UTILITY AND application of liver-specific MRI is expanding rapidly, creating new challenges and opportunities. Optimization of existing protocols and further technological developments to meet the growing demand in this field will improve the accuracy of, and increase the indications for, liver-specific MRI. In time, evolution of strategies for MRI with liver-specific contrast agents should positively impact patient management strategies in routine clinical practice.

Current challenges in liver-specific MRI, alongside the latest evidence in this field, were discussed at the 6th International Forum for Liver MRI, held in Vancouver, Canada, in September 2012, and attended by approximately 100 international abdominal radiologists, hepatologists, pathologists, and surgeons. The authors of this manuscript presented to the entire forum recent data in four key areas; these topics were then discussed further in breakout workgroups, each consisting of approximately 25 expert participants. The areas of discussion were: (i) Accurate assessment of liver function, (ii) Remaining challenges in metastatic liver disease, (iii) Remaining challenges in cirrhosis, and (iv) Hepatocellular carcinoma (HCC) management guidelines, the case for inclusion of liver-specific imaging.

Each workgroup generated and refined several consensus statements, with the aim to inform and advance current thinking in the subject areas, based on the experience and opinions of the expert participants. The consensus statements were then presented to the entire forum, refined according to further discussion, and voted on using an electronic voting system; the available options were "agree," "disagree," or "abstain." 
This manuscript summarizes the consensus of the forum attendees. The consensus statements are presented throughout the manuscript along with supporting evidence, and the number and percentage of liver forum participants who agreed with each statement are also reported. The authors' intent is that some consensus statements will be useful for radiologists in their daily clinical routine, while others will help to identify areas in which further research is needed.

It should be noted that this manuscript focuses on liver-specific MRI with gadoxetic acid. The focus on gadoxetic acid-enhanced MRI reflects the emphasis of the Liver Forum, in which scientific data were presented only for MRI performed with this agent. It is, therefore, beyond the scope of the current manuscript to provide comparisons of gadoxetic acid and other contrast agents used in liver-specific MRI. Some of the consensus statements may also apply to other liver-specific contrast agents.

\section{ACCURATE ASSESSMENT OF LIVER FUNCTION}

Postoperative mortality following liver resection has been decreasing over the last two decades, with many centers reporting mortality rates $<1 \%(1,2)$. Currently, the largest cause of postoperative mortality after liver resection is liver failure, and to avoid these fatalities, an accurate assessment of the future liver remnant (FLR) should be part of the preoperative work-up. The FLR must be of sufficient volume and quality to sustain postoperative liver function and enable regeneration.

In patients with normal liver function, the FLR is assessed in terms of volume measurements only, commonly using computed tomography (CT) or MRI (3). In patients with healthy liver parenchyma, a FLR $>20 \%$ of the total estimated functional liver volume is usually sufficient. However, worldwide opinions on the minimum required FLR volume vary in the range of $15 \%$ to $40 \%$ (4).

Many patients being considered for resection do not have normal liver parenchyma. This problem is common in patients with $\mathrm{HCC}$, with the majority of these tumors occurring in patients with cirrhosis and compromised liver function. As chemotherapy is used more frequently in multimodality treatment plans for patients with colorectal cancer liver metastases (CRCLM), chemotherapy-associated parenchymal dysfunction is increasingly encountered in this patient group (5), and has shown an association with increased morbidity, and in some cases even mortality, after resection. In patients with decreased liver function, the minimum volume of the FLR has to be increased according to the level of parenchymal damage and associated liver dysfunction (6).

Currently, evaluations of liver function fall into three main categories: routine laboratory tests measuring surrogate markers (serum bilirubin, albumin, coagulation factors, etc); scoring systems combining laboratory and clinical information (eg, ChildTurcotte-Pugh and Model for end-stage liver disease [MELD] scores); and quantitative liver function tests (eg, indocyanine green [ICG] clearance, albumin syn- thesis tests, LiMAx), which measure specific aspects of function (eg, microsomal or cytosol function, or hepatic membrane receptor expression) or hepatic perfusion (clearance of high extractable substances such as ICG, sorbitol, or galactose) $(7,8)$. However, the use of liver function tests for preoperative assessment of patients varies worldwide (4), and regional algorithms for HCC treatment strategies include different combinations of liver function assessment methods, with nonuniform adoption of guideline recommendations being common. For example, the European Association for the Study of the Liver - European Organisation for Research and Treatment of Cancer (EASL-EORTC) and Barcelona Clinic Liver Cancer (BCLC) practice guidelines endorse Child-TurcottePugh score, portal pressure, and bilirubin for patient staging (9), whereas guidelines from the Japan Society of Hepatology (JSH) suggest the use of the ChildTurcotte-Pugh score alone (10). Furthermore, local practices in many Asian centers include ascites, bilirubin and ICG measurements (11).

Current methods for assessing liver function, as outlined above, measure global function, with no consideration for segmental variations in function or dysfunction (ie, any reduction in liver function is assumed to be uniform throughout the parenchyma). However, in some conditions, liver function is not homogeneously distributed, with large segmental variations observed (12). Although postoperative mortality for patients undergoing liver resection is already low, global functional assessment methods may result in overly conservative decision-making, incorrectly denying some patients potentially curative surgical treatment. For example, if malignant lesions are located preferentially in areas of the liver with diminished function, while areas free of malignant lesions have more normal function, a larger amount of hepatic parenchyma may be resected safely than would be predicted based on global function tests. Thus, there is a need to develop more sophisticated methods to assess liver function at the segmental level.

\section{Imaging-Based Methods for Liver Function Assessment}

Imaging-based functional liver analyses have the potential to provide more accurate, multicompartmental measurements, including data on a multitude of liver function and physiology parameters within a single examination. Additionally, the ability to perform analyses on a segmental, or even subsegmental, level would allow clinicians to detect and account for any inhomogeneities in parenchymal function.

Hepatobiliary scintigraphic methods using radiopharmaceutical agents (technetium-99 nuclear isotope $\left[{ }^{99 m}\right.$ Tc]-labeled iminodiacetic acid, galactosyl serum albumin, or mebrofenin) have shown good correlation with ICG clearance, but are hampered by poor anatomical resolution (Fig. 1a) (13). To enable accurate segmental assessment, a tomographic method (single photon emission computed tomography [SPECT]) should be used, rather than a planar 

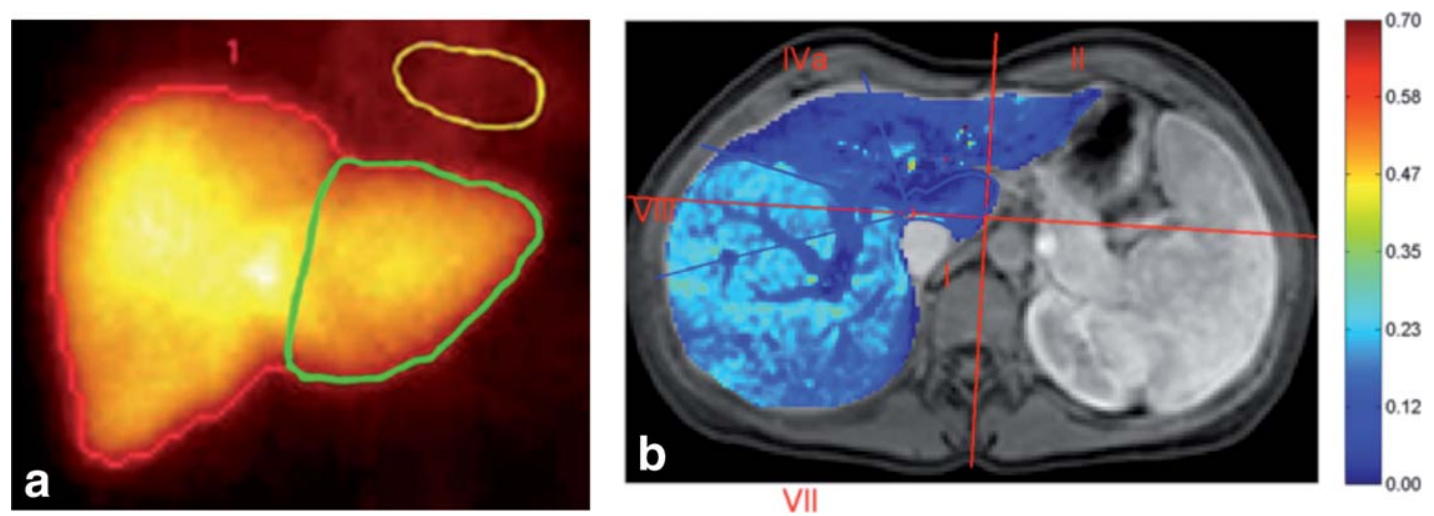

Figure 1. Imaging-based functional analyses of the liver: (a) hepatobiliary scintigraphy and (b) gadoxetic acid-enhanced MRI. a: Hepatobiliary CT scintigraphy with a radiopharmaceutical tracer provides a low resolution map of liver function, allowing lobular analysis (colored gating) (13). b: Hepatic extraction fraction MRI mapping of gadoxetic acid clearance provides high resolution anatomical images which can be interpreted at a segmental level. Part (a) reproduced with kind permission from Springer Science+Business Media: J Gastrointest Surg, Assessment of future remnant liver function using hepatobiliary scintigraphy in patients undergoing major liver resection, 2010;14:369-378, de Graaf W, et al, Part (b) reproduced with permission from Magnetic resonance imaging for the assessment of liver function, published by Karolinska Institutet. Henrik Nilsson, 2011, ISBN 978-91-7457-360-2.

imaging protocol (14). MRI is a multi-parametric technique that, apart from its use as diagnostic modality for detection and characterization of focal liver lesions and diffuse liver diseases, offers excellent anatomic resolution, useful for treatment planning and volumetry. In addition, physio-pathological information can be obtained by including MRI sequences, which enable elastography, relaxometry, diffusion-weighted imaging (DWI), and hepatobiliary phase (HBP) imaging, generating functional data regarding parenchymal architecture, fibrosis development, and hepatic dysfunction (15-17).

Gadoxetic acid is taken up by functioning hepatocytes using uptake and excretion receptor pathways common to other functional analysis agents (ICG, ${ }^{99 m}$ Tc-mebrofenin), namely, uptake by means of organic anion-transporting polypeptide (OATP) 1B1/ $\mathrm{B} 3$ and excretion through multi-drug resistance-associated protein 2 (MRP2) (18). Currently, an active area of investigation is the potential use of gadoxetic acidenhanced MRI to evaluate hepatic function, and several approaches have been explored (19-24).

The simplest approach is to perform static measurements of parenchymal enhancement during the HBP ( $\sim 20$ min after injection of gadoxetic acid). Mean relative enhancement of the parenchyma has been shown to correlate with the severity of cirrhosis (23) and stage of fibrosis (22), liver function (as assessed by surrogate marker measurements), and the risk of postoperative liver failure (19). The relative uptake of gadoxetic acid (signal intensity per liver volume) has also been shown to correlate with ICG clearance (24). The advantage of these static measurements is that they are simple to perform, without the necessity of additional sequences. However, the relationship between signal intensity and gadoxetic acid concentration is not linear, signal intensity can be confounded by several technical factors, and sampling error can occur according to region-of-interest (ROI) placement. A less subjective method that involves measurement of the absolute change in $\mathrm{T} 1$ or $\mathrm{T} 2 *$ relaxation with gadoxetic acid uptake has been shown to correlate with the Child-Turcotte-Pugh score $(20,21)$. This technique, however, involves additional scan sequences and dedicated software programs.

Hepatic uptake of gadoxetic acid occurs progressively over time, and a single time point measurement may be insufficient to reflect the enhancement profile or the hepatic perfusion. To address this, a more comprehensive approach involving dynamic hepatic gadoxetic acid-enhanced MRI has been investigated as a functional analysis tool. In this approach, multiple phases are acquired postinjection, and the parenchymal enhancement response over time in relation to a single vascular input, for example the portal vein, is plotted. A free deconvolution model then allows calculation of quantitative functional parameters, including the hepatic extraction fraction (HEF; amount of contrast agent that would be eliminated in one passage through the liver) and input relative blood flow (irBF; a measure of perfusion) (25), which can be depicted on high-resolution image maps (Fig. 1b). These dynamic measures of hepatic function, which can be assessed on segmental and subsegmental levels, are significantly reduced in disease, even in mild cirrhosis (26). Further refinement of this technique using a dual-input, dual-compartment model can improve combined functional and perfusion assessment, to better evaluate focal liver lesions and diffuse hepatic abnormalities (27). However, in their current form these dynamic acquisitions are time consuming and require dedicated software to complete the complex modeling, limiting their usefulness in clinical practice.

Dynamic hepatic gadoxetic acid-enhanced MRI as a method for assessment of liver function requires further development and validation to explore its potential and possible role in routine practice. However, because there is no "gold standard" against which to compare it, this is a problematic feat. In studies of this method as a decision-making tool regarding liver function assessment for patients considered for liver resection, survival should ideally be the primary endpoint. 
However, the number of patients needed to determine a significant survival-related outcome benefit in a randomized controlled trial would be prohibitively large, given the low mortality rates currently observed with hepatic resection (in one power analysis, it was estimated that 15,614 patients would be required to show a $33 \%$ relative reduction in operative mortality (28). Furthermore, the relatively high cost of gadoxetic acid-enhanced MRI dictates that no compromise in diagnostic quality should be made to obtain functional data. Dynamic, HBP, and functional sequences should be optimized to make "one-stop-shop" MRI assessment as accurate and cost-effective as possible. It is expected that recent technological developments in MR hardware, rapid sequences, and reconstruction techniques (such as compressed sensing) will make high spatial and temporal resolution T1-weighted imaging possible for "one-stop-shop" MRI anatomic and functional assessment of the liver. Confident extension of indications for surgery using functional MRI assessment, with maintenance of the current low mortality rates, should be the goal for the future.

Consensus statement 1.1: Given the limitations of current clinical parameters, there is a growing need to develop accurate and reproducible quantitative tools for regional liver function in patients being evaluated for liver resection. These tools have the potential to improve surgical decision-making and extend the indications for curative intended treatment $(77 / 85$ [90.6\%] agreement).

Consensus statement 1.2: Currently there is nonuniformity in the method used for liver function evaluation in patients who are surgical candidates. Comprehensive morphological and functional analysis of the liver using hepatocyte-specific contrastenhanced MRI shows potential that warrants further technical development and prospective clinical validation (79/84 [94.0\%] agreement).

\section{REMAINING CHALLENGES IN METASTATIC LIVER DISEASE}

The liver is a very common site of metastatic spread of cancer, in addition to lymph nodes, lungs, and bone; moreover, $30 \%$ to $70 \%$ of all cancer patients who die from their disease have liver metastasis (29). The most frequent primary cancers leading to liver metastasis are those of the colon (50\%), stomach, esophagus, pancreas, breast, lung, and eye (29).

\section{Imaging Features of Liver Metastases}

Metastatic lesion appearance is influenced by tumor growth and neo-arterial vascularity. Approximately two thirds of metastases demonstrate arterial phase hypoenhancement on dynamic imaging (hypovascular metastases), with the remainder showing arterial phase hyperenhancement (hypervascular metastases). Hypervascular metastases occur more commonly from primary pancreatic neuroendocrine or endocrine tumors, renal cell carcinoma, or breast cancer (30). Despite variable histologies and vascularity, metasta- ses share common imaging features. Circumferential arterial ring enhancement on dynamic contrastenhanced (CE) imaging is characteristic of metastases (positive predictive value [PPV], 82\%) (31); for hypervascular metastases, the ring corresponds to the enhancing edge of the tumor, while for hypovascular metastases it corresponds to peritumoral desmoplastic reaction, inflammatory cell infiltration, and vascular proliferation (32). High signal intensity is also commonly seen on DWI (33), attributable to restricted diffusion of water caused by hypercellularity (34). In the delayed phase, hypervascular metastases can show central hyperenhancement and peripheral hypoenhancement ( $\sim 30 \%$ cases); this "peripheral washout" pattern is especially common in patients with neuroendocrine and carcinoid tumors (35). Metastases that appear near isovascular to the liver parenchyma on CE-MRI also occur, especially following chemotherapy treatment.

\section{Challenge of Current Imaging Modalities}

CT and conventional MRI are the primary imaging modalities used for the evaluation of suspected liver metastases. A current challenge with metastatic disease is the characterization of subcentimeter, lowdensity lesions on CT; these lesions are often benign, but may be mucinous metastases, and a differential diagnosis is important for patient outcome.

CT and MRI have a similar, acceptable sensitivity for detection of metastases $>1 \mathrm{~cm}$ (87-89\%), but for lesions $<1 \mathrm{~cm}$ the sensitivity is much lower ( $60 \%$ for MRI and $\sim 47 \%$ for CT) (36). This low sensitivity hinders the selection of such patients for surgery and suboptimal management may occur, whereby patients with a large unrecognized metastatic burden may undergo unnecessary surgery, or patients with potentially resectable, yet undetected, metastases may undergo incomplete resection. Intraoperative ultrasound (US) can aid detection of lesions missed at preoperative imaging; however, this modality is $<100 \%$ sensitive and cannot aid preoperative patient selection or surgical planning. A technique that can accurately and noninvasively characterize metastases $<1 \mathrm{~cm}$ could improve appropriate patient selection for surgery and would aid the differential diagnosis of mucinous metastases.

\section{Gadoxetic Acid-Enhanced MRI for Imaging Metastases}

For visualization of metastatic disease, liver-specific MRI with gadoxetic acid has the advantage of a pseudo-steady-state HBP, whereby hepatocytes progressively uptake contrast agent over many minutes while metastases do not, providing a useful differential in relative enhancement (Fig. 2). In terms of imaging, this means that metastases tend to appear hypointense relative to the healthy parenchyma in the HBP. Additionally, due to the high relaxivity of gadoxetic acid, in the HBP (when the agent achieves a relatively high intra-hepatocellular concentration) the normal liver parenchyma enhances intensely, 


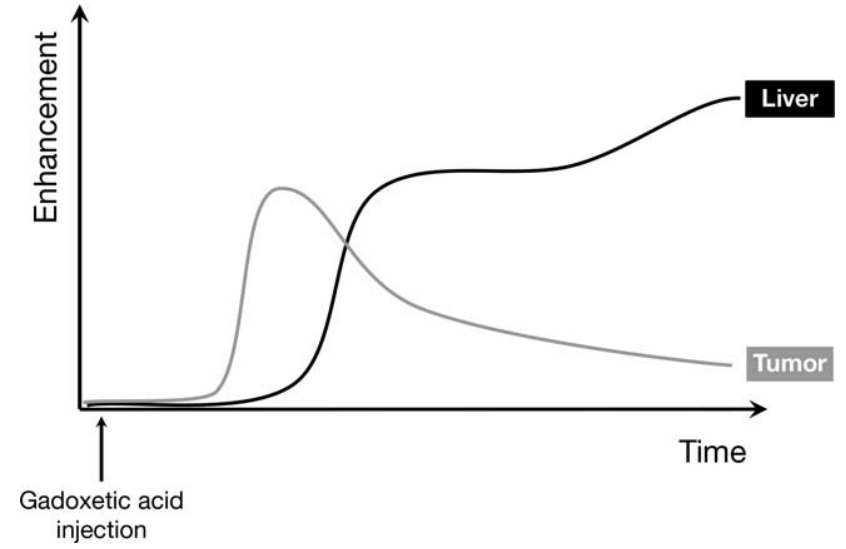

Figure 2. Schematic enhancement curves of a hypervascular tumor (eg, metastasis or HCC) and the progressive uptake of gadoxetic acid in the liver. Maximum enhancement in the liver occurs at approximately $15-30 \mathrm{~min}$ and is present in a pseudo-steady-state, permitting extended imaging times during the hepatobiliary phase using navigator-based or respiratory-triggered methods. This behavior of the delayed phase facilitates acquisition of very high spatial resolution images with high SNR/CNR performance.

providing high parenchymal signal-to-noise ratio (SNR) and high liver-to-lesion contrast-to-noise ratio (CNR). Also, because the HBP persists as a pseudosteady-state for many minutes, there is a prolonged window in which to acquire images, allowing high resolution, near isotropic images to be acquired (with multiple averages possible during free breathing using navigator sequences), while maintaining high SNR and CNR.

Added to dynamic phase images, HBP data can improve the detection of metastases. In a study of 107 metastases in 46 patients, gadoxetic acid-enhanced MRI including HBP images had a significantly higher area under the receiver operating characteristic curve and sensitivity compared with precontrast and postcontrast dynamic phase images alone $(0.91,97.2 \%$ versus $0.81,79.9 \%$, respectively; $P<0.05$ ) (37). Sensitivity was also higher in patients with small $(\leq 1 \mathrm{~cm})$ metastases and a history of chemotherapy $(91.6 \%$ including HBP images, compared with $48.6 \%$ for precontrast and dynamic postcontrast images only; $P<$ 0.001) (37).

As a modality, gadoxetic acid-enhanced MRI has demonstrated a higher sensitivity for focal liver lesion detection (67-96\%) than either nonenhanced MRI (46-84\%) or CT (61-85\%) $(38,39)$. Gadoxetic acidenhanced MRI was also more sensitive than tri-phasic CE-spiral CT for the detection of subcentimeter lesions (38.0-55.4\% versus 26.1-47.3\%) (38). For the detection of metastatic disease, gadoxetic acidenhanced MRI has demonstrated higher sensitivity than CE-multi-detector CT (MDCT); 96.4-97.3\% versus $76.4-80.9 \%$, respectively (40). Fifteen of 110 metastases were not detected using MDCT, but all of these lesions were identified on gadoxetic acidenhanced MRI (40). Additionally, the three metastases that could not be identified prospectively using either imaging modality, could be seen retrospectively to have subtle hypointensity in the HBP of gadoxetic acid-enhanced MRI (40). It is important to note that DWI was not performed in these studies. DWI is known to improve the detection of metastatic disease in the liver (41), and is complementary to gadoxetic acid-enhanced MRI for the detection and characterization of liver metastases (42); however, as discussed further below, gadoxetic acid-enhanced MRI alone may perform better than DWI alone (42).

Gadoxetic acid-enhanced MRI has also been shown to be more specific than nonenhanced MRI and CT in classification of benign versus malignant focal liver lesions (98\% versus 92\% and 88\%, respectively) (39) and more accurate than MDCT in differentiating metastases from cysts and hemangiomas (0.98 versus $0.89 ; P=0.0001)$ (40). This increased specificity was associated with improved diagnostic confidence $(39,40)$. To our knowledge, no literature evidence has compared dynamic extracellular contrast medium (ECCM)-enhanced MRI with liver-specific MRI for the evaluation of metastatic disease.

Although hypointensity in the HBP of gadoxetic acid-enhanced MRI may improve the detection of metastatic disease, this finding in isolation is not specific for metastases, being observed for several primary neoplasms (eg, HCC, hepatocellular adenoma, cholangiocellular carcinoma [CCC]) and benign lesions (eg, cysts and hemangiomas), as well as some pseudolesions (eg, focal parenchymal fat accumulation, perfusion alterations, parenchymal changes associated with chemotherapy, or locoablative therapy). Accurate characterization of HBP hypointense lesions requires assessment in the context of other available sequences, including T2-weighted images, DWI, and fatsensitive in- and opposed-phase images. DWI is well established as a useful method for detecting metastatic disease to the liver. Direct comparison of gadoxetic acid-enhanced MRI versus DWI alone has shown the superiority of gadoxetic acid-enhanced MRI for liver metastasis detection (42); however, these methods are highly complementary and the combination of DWI and gadoxetic acid-enhanced MRI shows great promise for the detection and characterization of liver metastases.

Consensus statement 2.1: The addition of hepatobiliary phase imaging to routine sequences improves the sensitivity for detection of metastatic disease to the liver (59/60 [98.3\%] agreement).

Consensus statement 2.2: Characterization of liver lesions depicted with hepatobiliary phase imaging must be performed in conjunction with routine sequences, to improve accuracy (59/60 [98.3\%] agreement).

\section{Optimization of the Imaging Protocol}

Because liver contrast enhancement (signal) is directly related to contrast agent relaxivity $\left(r_{1}\right)$ and parenchymal concentration [Gd] according to the following equation:

$$
\Delta \frac{1}{T_{1}}=\mathrm{r}_{1}[G d]
$$

for any given contrast agent tissue concentration, signal can be maximized by optimizing the T1-weighting. 

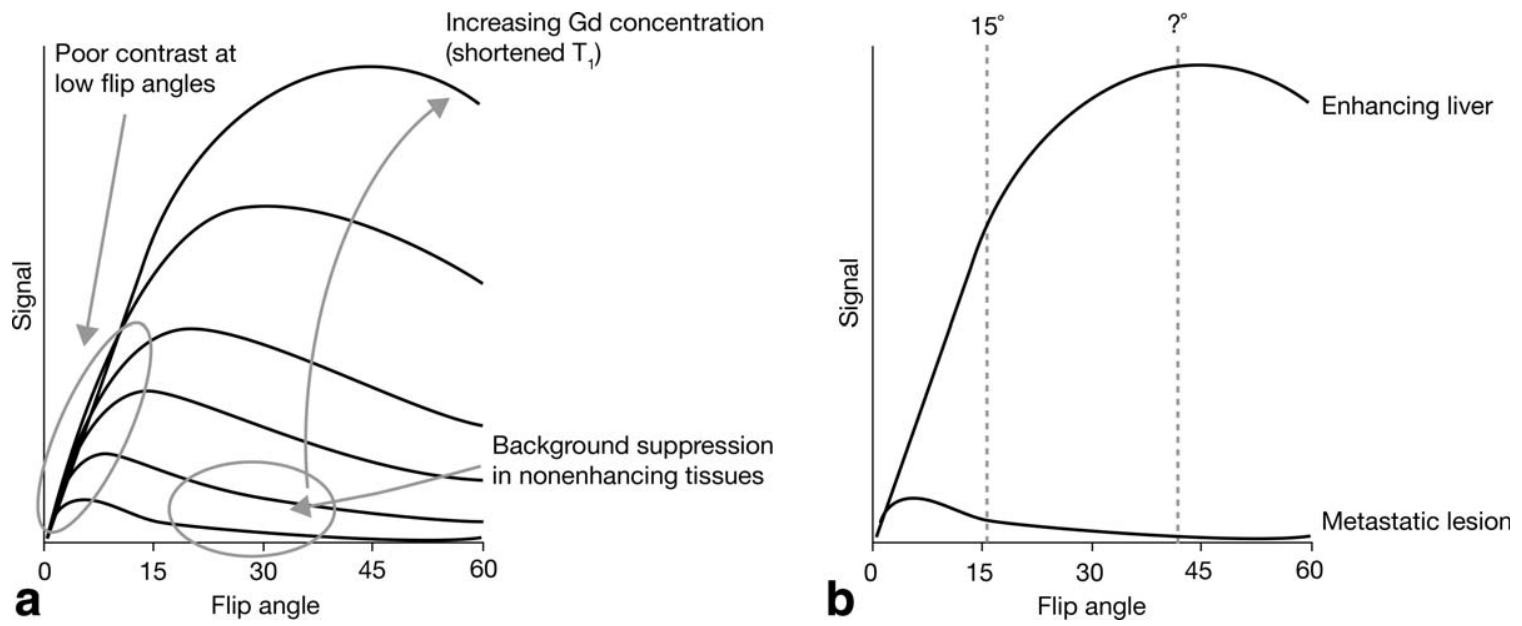

Figure 3. Schematic of the relationship between signal intensity in liver-specific CE-MRI and T1-weighting (flip angle) at increasing tissue concentrations of contrast agent (a), and schematic representation of relative signal intensity between parenchyma in the HBP (high contrast agent concentration in hepatocytes) and a metastatic lesion (no uptake of contrast agent) (b).

This can be achieved by selecting the optimal flip angle (Fig. 3a). In gadoxetic acid-enhanced MRI, a flip angle of $10^{\circ}$ to $15^{\circ}$ may be commonly applied for dynamic sequences, but during the HBP where hepatocyte concentration of gadoxetic acid is high, this is unlikely to provide optimal signal and relative liver-tolesion enhancement (Fig. 3b). Nagle et al demonstrated that for HBP imaging with gadoxetic acid, the optimal flip angle was around $40^{\circ}$, which provided twice the liver-to-muscle contrast compared with a $15^{\circ}$ flip angle (43). This increased relative enhancement can translate into improved lesion detection: in a comparison of low (10-12 ) and high (30-35') flip angles for HBP imaging of focal liver lesions in gadoxetic acid-enhanced MRI, the higher flip angle provided a $74 \%$ increase in lesion-to-liver contrast, which was associated with a higher detection rate for all lesions, especially small ones (sensitivity $81 \%$ versus $66 \%$ for low flip angle, $P<0.01$ ), an increased conspicuity score (especially for small lesions), and an increased detection rate for $\mathrm{HCC}$ (97\% versus 85\%) (44). In this study, conspicuity was increased for all types of lesions, but this did not specifically include metastases; it is expected that this will hold true for metastatic disease, although confirmatory studies are needed.

Most of the current evidence for T1 signal optimization in the HBP of liver-specific MRI is from healthy volunteers. In patients with reduced parenchymal function, hepatocyte uptake of gadoxetic acid and, therefore, relative enhancement versus lesions would be less. In patients with liver disease (eg, cirrhosis), signal optimization by means of flip angle adjustment may still be possible, but the optimal flip angle is likely to be lower than $40^{\circ}$, and further research is required to determine the best sequence parameters.

Consensus statement 2.3: The optimization of T1weighting for hepatobiliary phase imaging results in higher liver-to-lesion CNR and improves the conspicuity and detection rate of liver lesions (59/64 [92.2\%] agreement).

\section{REMAINING CHALLENGES IN CIRRHOSIS}

Gadoxetic acid-enhanced MRI has several advantages for noninvasive assessment of the cirrhotic liver, including higher sensitivity for the diagnosis of HCC, especially for lesions $\leq 2 \mathrm{~cm}$ (45-49); improved characterization of arterially enhancing lesions without definite "washout" (47,50); distinguishing arterially enhancing pseudolesions from $\operatorname{HCC}(50,51)$; and detection of lesions that are isointense to the parenchyma on all sequences apart from the HBP, and that are at high risk of transforming to hypervascular HCC $(52,53)$. However, some limitations to the use of gadoxetic acid-enhanced MRI in the cirrhotic liver remain under discussion.

In some patients, particularly those with advanced cirrhosis (Child-Turcotte-Pugh score B and C), gadoxetic acid-enhanced MRI can show weak parenchymal and venous enhancement $(54,55)$, which may hamper the detection of $\mathrm{HCC}$ and the assessment of portal vein patency. A simple clinical measure to predict reduced gadoxetic acid enhancement at MRI could allow more appropriate selection of patients in whom gadoxetic acid-enhanced MRI is likely to be beneficial (versus those in whom an extracellular agent may be more appropriate).

Studies investigating clinical markers of liver function as predictors of enhancement with gadoxetic acid have shown variable findings. Routine clinical parameters such as aspartate aminotransferase (AST), total bilirubin, albumin, and prothrombin activity have shown correlations with gadoxetic acid enhancement in some studies (56-60), although these were not always predictive factors. Total bilirubin has been used as a selection criterion in some centers, but the cutoff value (between 2 and $3 \mathrm{mg} / \mathrm{dL}$ ) is somewhat arbitrary, and other causes of hyperbilirubinemia (eg, Gilbert's syndrome) exist which are not associated with parenchymal dysfunction. ICG clearance has shown variable degrees of association with gadoxetic acid enhancement $(58,61,62)$. MR elastography as a 
measure of parenchymal stiffness was predictive in one study (60), but elastography is not likely to be a clinically feasible method for determining the suitability of gadoxetic acid-enhanced MRI. A relatively consistent association with reduced gadoxetic acid uptake has been stage of cirrhosis, especially more severe disease (Child-Turcotte-Pugh score C) $(23,59,61,63)$. MELD scoring may be a more objective assessment of liver function than the older ChildTurcotte-Pugh score, and it correlates with mortality. Early reports also suggest a correlation between the MELD score and intensity of delayed parenchymal and biliary enhancement on gadoxetic acid-enhanced MRI $(64,65)$. In principle, the most reliable markers for gadoxetic acid uptake should be hepatic expression of the transporters OATP 1B1/B3 and MRP2 $(66,67)$. However, evaluation of this expression involves immunohistochemical staining of biopsy or surgical tissue, which would typically be unavailable, as few centers perform routine immunohistological analysis for these proteins. More research is needed into the correlations between OATP 1B1/B3 and MRP2 expression, gadoxetic acid uptake, and markers of liver function, to translate the evidence into meaningful clinical practice that can improve patient management.

Consensus statement 3.1: Gadoxetic acid-enhanced MRI in some patients with cirrhosis can show weak lesion-to-liver contrast in all phases and poor venous enhancement, which may hamper the diagnosis of HCC and assessment of portal vein patency. Multicenter studies to determine the correlation between enhancement at gadoxetic acid-enhanced MRI and markers of liver function are required (66/77 [85.7\%] agreement).

Infrequently, nodules that are isointense to the parenchyma in the dynamic phases and hyperintense in the HBP of gadoxetic acid-enhanced MRI are discovered, and these nodules may also appear isointense on T2-weighted sequences. Limited data from the literature suggest that the vast majority of such lesions are benign, regenerative or hyperplastic nodules (fed by the portal vein), or focal nodular hyperplasia (FNH)-like lesions (68). Progression to a hypervascular nodule has been described rarely $(<1 \%$ cases) $(69,70)$, although arterial hypovascularity may signify a risk for malignancy (high-grade dysplastic nodules [DNs] or early HCC).

Consensus statement 3.2: Nodules with arterial hypo-/isovascularity, venous isovascularity and HBP hyperintensity are likely to be benign; however, the role of size in predicting the outcome of these nodules is still not clear (56/75 [74.7\%] agreement)

\section{Hypo-/Isovascular, HBP Hypointense Nodules}

Hypointensity in the HBP of gadoxetic acid-enhanced MRI is suggestive of premalignancy or malignancy, independently of vascular enhancement pattern; indeed, almost all HCC and some high-grade DNs are hypointense in this phase (71). Therefore, gadoxetic acid-enhanced MRI has increased the detectability of small hypo- or isovascular premalignant or early HCC ("borderline") lesions (47), which are common in cirrhotic patients. The detection of such nodules is important, because studies have shown that vascular invasion, metastatic spread, and recurrence after radiofrequency ablation are less frequent in hypovascular versus hypervascular small $\operatorname{HCC}(72,73)$. While some low-grade DNs and regenerative nodules (RN) also show hypointensity in the $\operatorname{HBP}(47,74)$, these nodules are usually $<1 \mathrm{~cm}$ (75), while high-grade DN and early HCC tend to be $>1 \mathrm{~cm}$. Therefore, a hypovascular or isovascular, HBP hypointense nodule of $>1 \mathrm{~cm}$ in at-risk patients would strongly suggest a premalignant or malignant lesion.

The prognosis of hypovascular or isovascular "borderline" nodules in cirrhotic patients has been studied in several centers. Kumada et al reported that $13 / 49$ (26.5\%) of nodules developed arterial hypervascularity during the follow-up period (3-15 months, median 6 months), and these lesions showed a greater change in size than nonvascularized lesions (53). Size of nodule was found to be a significant factor for prognosis, with a 1-year hypervascularization risk of $17 \%$ for nodules $<1.5 \mathrm{~cm}$ compared with $77 \%$ for those $\geq 1.5 \mathrm{~cm}(P=0.0147)$ (53). Another study by Motosugi et al reported arterial vascularization in 16/135 (11.9\%) nodules, with a median time to hypervascularization of 288 days (median follow-up duration, 296 days) (76). Size was again related to hypervascularization, with a 1 -year risk of $37.6 \%$ for nodules $>1 \mathrm{~cm}$, compared with $15.6 \%$ for all nodules. A receiver operating characteristic (ROC) analysis revealed an optimal cutoff in nodule size to predict hypervascularization of $1.1 \mathrm{~cm} \mathrm{(76).} \mathrm{Presence} \mathrm{of} \mathrm{fat}(P<0.01)$, enlargement during follow-up $(P=0.04)$ and T2 hyperintensity $(P=$ 0.06) were also associated with an increased risk of hypervascularization (76). Akai et al further demonstrated a relationship between initial nodule size and hazard ratio for HCC development, based on 17/130 (13.1\%) nodules that developed hypervascularity (77). The cumulative rate for development of classic HCC was $3.2 \%$ at 1 year, and $11.1 \%$ and $15.9 \%$ after 2 and 3 years, respectively. Takayama et al performed a multivariate analysis which showed that a nodule size of $\geq 0.9 \mathrm{~cm}$ was a significant predictive factor for malignant transformation (enlargement and/or vascularization; $P<0.05)$ (78).

Therefore, while hypo-/isovascular, HBP hypointense nodules smaller than $1 \mathrm{~cm}$ have some risk for malignant transformation, the risk appears to be substantially greater for larger nodules. The strong association between nodule size and tumor transformation, and the poorer prognosis following hypervascularization (increased invasion, spread, and recurrence), suggests that these "borderline" lesions may warrant expedited follow-up, assessment using other imaging modalities or biopsy and/or consideration for treatment; however, the optimal management strategy is, as yet, unknown and more research is needed.

Consensus statement 3.3: Cirrhosis-associated nodules $>1 \mathrm{~cm}$ with arterial hypo-/isovascularity and HBP hypointensity may have a high risk of becoming hypervascular HCC within 1 year (59/74 [79.7\%] agreement). 
Consensus statement 3.4: Features that suggest a higher likelihood of developing hypervascular HCC may include lesion size, lesion growth, T2 hyperintensity, and intralesional fat. Further studies are needed to confirm these and other potential predictors $(64 / 72$ [79.7\%] agreement).

Consensus statement 3.5: Arterial hypovascular/ isovascular, HBP hypointense nodules $>1 \mathrm{~cm}$ in diameter can be considered for biopsy or additional/closeinterval imaging follow-up (60/65 [92.3\%] agreement).

\section{HCC MANAGEMENT GUIDELINES: THE CASE FOR INCLUSION OF LIVER-SPECIFIC IMAGING}

Currently, only one set of guidelines for the management of HCC includes liver-specific MRI in its diagnostic algorithm: in its 2010 guidelines update the JSH recommends gadoxetic acid-enhanced MRI to further investigate nodules that have not shown a clear arterial phase hyperenhancement and portal venous or delayed phase "washout" pattern on a dynamic imaging technique (CE-CT, -MRI, or -US) (10).

Other recently updated guidelines from the EASLEORTC (9) and the American Association for the Study of Liver Diseases (AASLD) (79) make no contrast agent recommendations. These guidelines both suggest that suspected $\mathrm{HCC}<1 \mathrm{~cm}$ should receive follow-up imaging with US, while nodules $>1 \mathrm{~cm}$ should be investigated by MDCT or dynamic CE-MRI for the characteristic arterial phase hyperenhancement and venous/delayed phase "washout" pattern. If this pattern of contrast enhancement is not observed using the initial dynamic imaging modality, the alternative modality or biopsy is recommended. If a second modality is used and also equivocal, biopsy is recommended. However, these guidelines make no suggestion as to which modality should be the initial imaging method.

In deciding on the initial imaging modality for further investigation of suspected HCC, the accuracy of MDCT, ECCM-MRI, and gadoxetic acid-enhanced MRI for the diagnosis of HCC should be considered. To our knowledge, there has been only one direct comparison reported between gadoxetic acid-enhanced MRI and ECCM-MRI for the detection of HCC, in which gadoxetic acid was associated with a significantly higher sensitivity versus gadopentetate dimeglumine $(86.4 \%$ versus $64.4 \%$, respectively) for detecting small HCC $(0.5-2 \mathrm{~cm})$ and a trend toward higher area under the receiver operator characteristic curve (0.958 versus 0.927 , respectively), which did not reach significance $(P=0.362)(80)$. Compared with MDCT, gadoxetic acid-enhanced MRI appears to have similar sensitivity and accuracy for larger HCC $(>1.5 \mathrm{~cm})$, but may show an improvement over MDCT for smaller nodules $(48,49,81,82)$.

Consensus statement 4.1: The reported accuracy of gadoxetic acid-enhanced MRI for diagnosis of HCC compares favorably with that of MDCT and MRI performed with extracellular agents (ECCM-MRI). Hence, for the primary diagnosis of $\mathrm{HCC}$, management guide- lines should permit use of gadoxetic acid-enhanced MRI as an alternative to multi-phasic CT or ECCMMRI (50/60 [83.3\%] agreement).

A recent article highlighted that noninvasive imaging strategies for $1-3 \mathrm{~cm}$ nodules, based on the EASL and AASLD guidelines, required a second imaging modality in $50 \%$ of cases, and that at least $10 \%$ of HCC are missed completely (83). Additionally, it has been reported that only $61 \%$ of $\mathrm{HCC}$ nodules show hypervascularity according to the EASL criteria (44\% of 1-2 cm nodules), with $20 \%$ of nodules being hypovascular in two imaging techniques (84). Therefore, it is hypovascular HCCs that present a particular diagnostic challenge.

As discussed in section 3, HBP imaging in gadoxetic acid-enhanced MRI aids the identification of hypovascular premalignant and malignant lesions in the cirrhotic liver, due to the added information gained in this phase. Early HCC and high-grade DN, which largely appear hypointense to the parenchyma in this phase, can be distinguished from low-grade DN and $\mathrm{RN}$, which tend to be iso- or hyperintense. Compared with just the dynamic phase images, addition of the HBP data in a study of gadoxetic acid-enhanced MRI increased the sensitivity to identify and characterize small hypovascular HCC $(\leq 2 \mathrm{~cm})$ from $88.4 \%$ to $99.4 \%$, and also improved the specificity, accuracy, and positive and negative predictive values $188 \%$ to $95 \%$; $88 \%$ to $98.5 \%$; $97 \%$ to $99 \%$; and $65 \%$ to $97.5 \%$, respectively) (47). Furthermore, 19/20 atypical nodules (those not showing arterial hypervascularity and portal venous/delayed phase "washout") were characterized only during the HBP (47). Therefore, if a lesion can be detected on gadoxetic acid-enhanced MR images (ie, if it does not appear isointense), it can be more accurately characterized. This suggests that if gadoxetic acid-enhanced MRI is chosen as the initial imaging modality for further investigation of suspected HCC, a second modality may be less frequently required.

Consensus statement 4.2: With regard to indeterminate, iso-/hypovascular nodules $>1 \mathrm{~cm}$ identified with MDCT or dynamic CE-MRI:

- Current evidence supports the incorporation into management guidelines of hepatobiliary imaging with gadoxetic acid-enhanced MRI for possible further evaluation of such nodules (43/54 [79.6\%] agreement).

The current BCLC algorithm for early- and very early-stage HCC recommends the size and number of tumors as parameters to consider when reaching a treatment decision (85). A retrospective analysis of liver transplant patients has shown a linear relationship between size of the largest $\mathrm{HCC}$ and tumor recurrence, but a weaker association between the number of tumors and recurrence (86), suggesting that accuracy in measuring tumor diameter is the more important consideration. There are no data available showing that gadoxetic acid-enhanced MRI provides more accurate measurement of tumor size or number than either CT or ECCM-MRI. Treatment decisions for advanced stage HCC according to the BCLC algorithm should involve evaluation of vascular 
invasion and extra-hepatic spread (85); again, there is no evidence in the literature that gadoxetic acidenhanced MRI provides better evaluation of vascular invasion than other imaging modalities. Therefore, with respect to reaching a treatment decision on $\mathrm{HCC}$, further evidence is required to recommend inclusion of gadoxetic acid-enhanced MRI into the guidelines.

With regard to treatment response, the modified Response Evaluation Criteria in Solid Tumors (mRECIST) specify that complete response should be based on disappearance of tumoral enhancement in all target lesions (87). Hwang et al demonstrated that for evaluation of the posttreatment viability of $\mathrm{HCC}$, gadoxetic acid-enhanced MRI showed higher diagnostic accuracy than MDCT (96.3\% versus 80.9-82.7\%, respectively) and better sensitivity (92.9-96.4\% versus $53.6 \%$, respectively) (88). The negative predictive value was also higher for gadoxetic acid-enhanced MRI (96.3-98.1\% versus 79.5-80.0\% for MDCT) (88). However, we are not aware of any studies comparing gadoxetic acid-enhanced MRI and ECCM-MRI to assess treatment response, and to consider recommending the inclusion of gadoxetic acid-enhanced MRI into treatment-response guidelines, further data are necessary.

As discussed previously, the finding of hypointensity in the HBP of gadoxetic acid-enhanced MRI increases diagnostic confidence for premalignant or malignant lesions; however, to recommend inclusion of gadoxetic acid-enhanced MRI in HCC management guidelines, it must be shown that HCC can be adequately differentiated from other non-HCC malignancy, such as CCC, using this modality. Mass-forming intrahepatic CCCs frequently appear as noncapsulated, lobulated tumors, enabling differentiation from the round shape of classic HCCs (89). However, as HCC may also have a lobulated appearance, further gadoxetic acidenhanced MRI features should be considered. Massforming CCCs typically show peripheral enhancement in the early arterial phase, and progressive concentric (centripetal) enhancement in the portal and delayed phases, although atypical hypervascular massforming CCCs with complete or partial arterial enhancement and portal/delayed phase "washout" occur in up to $47 \%$ of cases (90). The differentiation of mass-forming intrahepatic CCC from hypovascular HCC may be difficult. A recent study suggests that signal intensity on T2-weighted images may help in the differentiation; in univariate analyses, the authors found that the presence of hypo- or hyperintense areas favors a diagnosis of CCC, while the presence of linear hyperintensity or multifocal, tiny hyperintense foci favors hypovascular HCC (89). In a multivariate analysis, however, the authors found that the only independent imaging feature on gadoxetic acid-enhanced MRI that differentiates mass-forming CCCs from hypovascular HCC was a central enhancing region with a peripheral hypointense rim (a so-called "target" appearance) on 10-minute HBP images (89), attributable to intratumoral fibrous tissue. However, caution is required in application of these findings, because scirrhous HCCs (which have abundant fibrous stroma) can show near identical imaging features to massforming CCCs (89). One imaging feature that may favor the diagnosis of scirrhous HCC is the presence of strong, thick peripheral arterial enhancement (89), although confirmatory studies are needed.

Consensus statement 4.3: For management guidelines to recommend gadoxetic acid-enhanced MRI as the primary technique for diagnosis of $\mathrm{HCC}$, prospectively designed studies are needed to show the superiority of gadoxetic acid-enhanced MRI over MDCT and ECCM-MRI in per-lesion accuracy for the diagnosis of HCC AND noninferiority for both of the following additional performance characteristics: (i) Per-patient accuracy for staging, including determination of size and number of HCCs, as well as presence of macrovascular invasion; (ii) Per-lesion specificity/PPV in differentiating HCC from non-HCC malignancy such as CCC (46/54 [85.2\%] agreement).

\section{Toward an Interpretation Guideline for Gadoxetic Acid-Enhanced MRI in Patients at Risk for HCC}

Regardless of the recommendation for gadoxetic acidenhanced MRI in HCC management guidelines, many centers already use this imaging modality in their routine clinical investigation of liver tumors. While gadoxetic acid-enhanced MRI is used frequently, the criteria applied for image assessment appear to vary widely, and an interpretation guideline for this technique may be warranted.

A systematic literature search was carried out to identify evidence for the predictive value of different gadoxetic acid-enhanced MRI temporal enhancement patterns in diagnosing hypervascular HCC in at-risk patients. The "at-risk" population largely consists of cirrhosis patients, but it also includes Asian chronic hepatitis B patients without cirrhosis, as well as patients with nonalcoholic fatty liver disease and advanced fibrosis but without cirrhosis. The search criteria were as follows, applied to studies published between 1990 and 2012 in English and involving humans: "(hepatocellular carcinoma OR hepatoma OR HCC) AND (magnetic resonance imaging OR MRI OR MR OR MR imaging OR NMRI) AND (eovist OR primovist OR gadoxetic acid OR gadoxetate OR gadoxetate disodium OR Gd-EOB OR Gd-EOB-DTPA OR EOB-DTPA) NOT (metastasis OR metastases)." Thirtyfour studies were identified that contained either explicit or extractable data on either (a) PPV for HCC diagnosis or (b) presence of false positives $(45-50,52-$ $54,76,77,80,81,89,91-110)$. The denominators for calculating PPVs were not consistently supplied in these manuscripts; however, the expert consensus of the HCC management guidelines workgroup, and of the entire forum, was considered in addition to the published evidence. A challenge in performing the systematic review was the inconsistent terminology and criteria used by different published studies; 19 of the identified manuscripts applied 16 individual criteria for diagnosing HCC on gadoxetic acid-enhanced MRI, and 14 different sets of these criteria.

Four temporal enhancement patterns for hypervascular lesions could be analyzed (Fig. 4). Most papers 


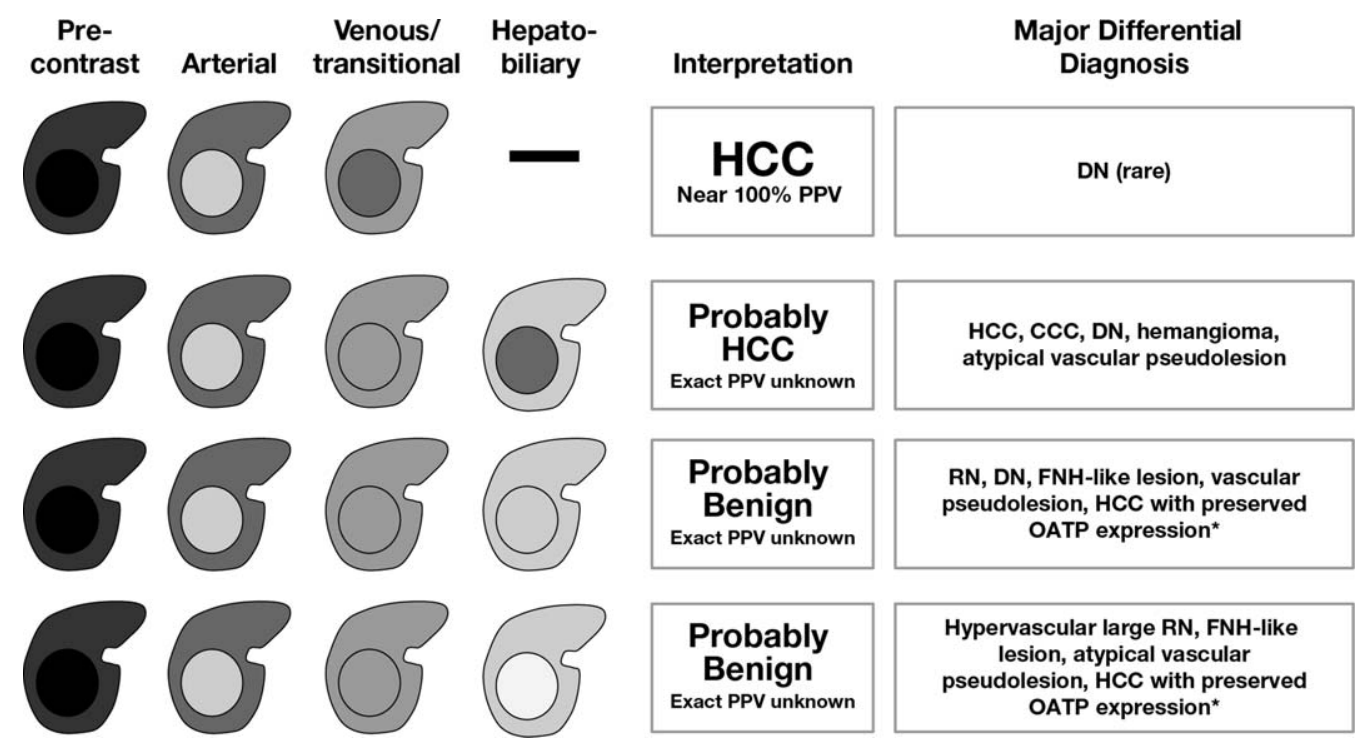

Figure 4. Provisional interpretation guide for enhancement patterns in the imaging of hypervascular HCC using gadoxetic acid-enhanced MRI. Schematic diagram illustrates, for lesions with arterial phase hyperenhancement, four different temporal enhancement patterns at gadoxetic acid-enhanced MRI. Most published studies have not differentiated between the venous and transitional phases; hence, these are considered together. For each pattern, a provisional interpretation based on published evidence and expert opinion is suggested. Entities representing a differential diagnosis are listed. *Suggestive of HCC with preserved OATP expression: focal uptake defects, heterogeneity, nodule-in-nodule appearance, absence of central scar, hypointense rim. CCC, cholangiocellular carcinoma; DN, dysplastic nodule; FNH, focal nodular hyperplasia; HCC, hepatocellular carcinoma; OATP, organic anion-transporting polypeptide; PPV, positive predictive value; RN, regenerative nodule.

did not distinguish between the portal venous and transitional phases; therefore, for the systematic review, these phases were considered together.

A pattern consisting of arterial phase hyperenhancement with venous/transitional phase hypoenhancement had a reported or extracted PPV of $100 \%$ for $\geq 1 \mathrm{~cm} \operatorname{HCC}(46,47,50,54,80,91,93,95,108)$. Only one published study reported a false positive finding for lesions with this pattern (a $0.9 \mathrm{~cm} \mathrm{DN}$ ) (98). Most studies considered HBP signal intensity as noncontributory for establishing the diagnosis of HCC for nodules or masses exhibiting this pattern. However, when no "washout" is seen in the venous/transitional phase (ie, the nodule appears isointense to the parenchyma), the signal intensity in the HBP is an important factor to help reach a diagnosis, as described below.

A lesion demonstrating arterial phase hyperenhancement, venous/transitional phase isoenhancement and HBP hypoenhancement in at-risk patients is most likely to be $\operatorname{HCC}(48,77,81,93,100,102,103,105)$, but the exact PPV could not be extracted from the selected studies. However, based on published reports and the experience of the liver forum participants, it is known that, in at-risk patients, lesions with this temporal enhancement pattern are not always HCC. Alternative diagnoses for nodular lesions showing this enhancement pattern are non-HCC malignancies such as intrahepatic CCCs, premalignant lesions like high-grade DNs (96), nonmalignant lesions including hemangiomas $(48,94)$ and DNs (98), and benign pseudolesions such as arterioportal shunts (Fig. 4) $(48,50,97,109)$. In this circumstance, ancillary imaging features may be useful. Ancillary features that, if present, may favor the diagnosis of HCC include intralesional fat (105), a capsule appearance (48), corona enhancement, and mosaic architecture (47). Other ancillary features favor malignant over benign entities, but are not specific for $\mathrm{HCC}$ as these features may also occur in CCCs and combined HCC-CCC tumors; these features include mild-moderate T2 hyperintensity $(45,54,80,93,101)$, DWI hyperintensity and, if hemangioma is excluded based on other features, lesional iron sparing. Conversely, size discrepancy between the arterial and HB phases (97), and irregular shape (101), may favor the diagnosis of a vascular pseudolesion. Despite these differential diagnostic considerations, forum participants from Japan indicated that some centers in Japan consider a nodule with arterial hyperenhancement, venous/transitional phase isoenhancement, and HBP hypoenhancement on gadoxetic acid-enhanced MRI as diagnostic for HCC in at-risk patients; this pattern is also considered diagnostic of HCC in the 2010 updated clinical practice guidelines endorsed by the JSH (10).

Consensus statement 4.4: In patients at risk for $\mathrm{HCC}$ imaged with gadoxetic acid-enhanced MRI:

a) $>1 \mathrm{~cm}$ arterial phase hyperenhancing nodules/ masses with portal venous phase/transitional phase hypoenhancement are diagnostic for HCC (50/57 [87.7\%] agreement).

b) $>1 \mathrm{~cm}$ arterial phase hyperenhancing nodules/ masses without portal venous phase/transitional phase hypoenhancement but with HBP hypointensity are highly suspicious, but not diagnostic, for HCC (57/58 [98.3\%] agreement).

(The term "diagnostic" is used here as a radiology criterion, with the understanding that some degree of uncertainty can never be completely eliminated). 
In at-risk patients, most lesions showing arterial phase hyperenhancement, venous/transitional phase isoenhancement and either HBP isoenhancement or HBP hyperenhancement are benign. While some of these lesions may represent HCC with preserved OATP expression, the majority are likely to be $\mathrm{RN}$, DN, FNH-like lesions or pseudolesions (Fig. 4). The PPVs for these enhancement patterns, however, are currently unknown. Ancillary multiparametric features may aid in the differential diagnosis of lesions with these patterns. Because non-HCC malignancies are not considered in the major differential diagnosis for such lesions, any ancillary feature suggestive of malignancy favors the diagnosis of $\mathrm{HCC}$, including features that in other circumstances may not be specific for HCC: mild-to-moderate T2 hyperintensity, DWI hyperintensity, lesional iron sparing, intralesional fat, a capsule appearance, and corona enhancement. Additionally, for nodules demonstrating HBP iso- or hyperintensity, HBP features that suggest a diagnosis of HCC are focal defects in uptake (110), heterogeneity $(77,81,93,99)$, a nodule-in-nodule or mosaic architecture (47), absence of a central scar (110), presence of internal septation(s) (110), or a hypointense rim (110).

\section{SUMMARY}

Gadoxetic acid-enhanced MRI shows potential for the combined morphological and functional analysis of the liver at the segmental level. This imaging modality also provides accurate detection of metastatic liver disease, with further improvements possible through protocol optimizations. In the cirrhotic liver, gadoxetic acid-enhanced MRI fosters diagnostic confidence for the characterization and management of several nodules, including "borderline" (arterial hypo-/isovascular HBP hypointense) lesions. Gadoxetic acidenhanced MRI performs favorably against CT and ECCM-MRI for the diagnosis of HCC, but to be recommended in HCC management guidelines as the primary diagnostic technique, more data are needed. An interpretation guideline for the assessment of gadoxetic acid-enhanced MRI images may be warranted to improve standardization within and between centers.

\section{ACKNOWLEDGMENTS}

The Sixth International Forum for Liver MRI was supported by Bayer HealthCare, and all authors received honoraria and travel expenses.

\section{REFERENCES}

1. Belghiti J, Hiramatsu K, Benoist S, Massault PP, Sauvanet A, Farges O. Seven hundred forty-seven hepatectomies in the 1990s: an update to evaluate the actual risk of liver resection (abstract). J Am Coll Surg 2000;191:38-46.

2. Jarnagin WR, Gonen M, Fong Y, et al. Improvement in perioperative outcome after hepatic resection: analysis of 1,803 consecutive cases over the past decade. Ann Surg 2002;236:397-406.

3. Karlo C, Reiner CS, Stolzmann P, et al. CT- and MRI-based volumetry of resected liver specimen: comparison to intraoperative volume and weight measurements and calculation of conversion factors (abstract). Eur J Radiol 2010;75:e107-e111.
4. Breitenstein S, Apestegui C, Petrowsky H, Clavien PA. "State of the art" in liver resection and living donor liver transplantation: a worldwide survey of 100 liver centers. World J Surg 2009;33:797-803.

5. Zorzi M, Barca A, Falcini F, et al. Screening for colorectal cancer in Italy: 2005 survey. Epidemiol Prev 2007;31:49-60.

6. Guglielmi A, Ruzzenente A, Conci S, Valdegamberi A, Iacono C. How much remnant is enough in liver resection? Dig Surg 2012; 29:6-17.

7. Seyama Y, Kokudo N. Assessment of liver function for safe hepatic resection. Hepatol Res 2009;39:107-116.

8. Morris-Stiff G, Gomez D, Prasad R. Quantitative assessment of hepatic function and its relevance to the liver surgeon. J Gastrointest Surg 2009;13:374-385.

9. European Association for the Study of the Liver,European Organisation for Research and Treatment of Cancer. EASLEORTC Clinical Practice Guidelines: management of hepatocellular carcinoma (abstract). J Hepatol 2012;56:908-943.

10. Kudo M, Izumi N, Kokudo N, et al. Management of hepatocellular carcinoma in Japan: consensus-based clinical practice guidelines proposed by the Japan Society of Hepatology (JSH) 2010 updated version. Dig Dis 2011;29:339-364.

11. Makuuchi M, Kokudo N, Arii S, et al. Development of evidencebased clinical guidelines for the diagnosis and treatment of hepatocellular carcinoma in Japan. Hepatol Res 2008;38:37-51.

12. Abdalla EK, Denys A, Chevalier P, Nemr RA, Vauthey JN. Total and segmental liver volume variations: implications for liver surgery (abstract). Surgery 2004;135:404-410.

13. de Graaf W, van Lienden KP, Dinant S, et al. Assessment of future remnant liver function using hepatobiliary scintigraphy in patients undergoing major liver resection. J Gastrointest Surg 2010;14:369-378.

14. Bennink RJ, Tulchinsky M, de Graaf W, Kadry Z, van Gulik TM. Liver function testing with nuclear medicine techniques is coming of age (abstract). Semin Nucl Med 2012;42:124-137.

15. Bonekamp S, Kamel I, Solga S, Clark J. Can imaging modalities diagnose and stage hepatic fibrosis and cirrhosis accurately? (abstract). J Hepatol 2009;50:17-35.

16. Heye T, Yang SR, Bock M, et al. MR relaxometry of the liver: significant elevation of $\mathrm{T} 1$ relaxation time in patients with liver cirrhosis. Eur Radiol 2012;22:1224-1232.

17. Tam HH, Collins DJ, Wallace T, Brown G, Riddell A, Koh DM. Segmental liver hyperintensity in malignant biliary obstruction on diffusion weighted MRI: associated MRI findings and relationship with serum alanine aminotransferase levels. Br J Radiol 2012;85:22-28.

18. Van Beers BE, Pastor CM, Hussain HK. Primovist, eovist: what to expect? (abstract). J Hepatol 2012;57:421-429.

19. Cho SH, Kang UR, Kim JD, Han YS, Choi DL. The value of gadoxetate disodium-enhanced MR imaging for predicting posthepatectomy liver failure after major hepatic resection: a preliminary study. Eur J Radiol 2011;80:e195-e200.

20. Katsube T, Okada M, Kumano S, et al. Estimation of liver function using T1 mapping on Gd-EOB-DTPA-enhanced magnetic resonance imaging. Invest Radiol 2011;464:277-283.

21. Katsube T, Okada M, Kumano S, et al. Estimation of liver function using T2* mapping on gadolinium ethoxybenzyl diethylenetriamine pentaacetic acid enhanced magnetic resonance imaging. Eur J Radiol 2012;81:1460-1464.

22. Motosugi U, Ichikawa T, Oguri M, et al. Staging liver fibrosis by using liver-enhancement ratio of gadoxetic acid-enhanced MR imaging: comparison with aspartate aminotransferase-to-platelet ratio index. Magn Reson Imaging 2011;29:1047-1052.

23. Tamada T, Ito K, Higaki A, et al. Gd-EOB-DTPA-enhanced MR imaging: evaluation of hepatic enhancement effects in normal and cirrhotic livers. Eur J Radiol 2011;80:e311-e316.

24. Yamada A, Hara T, Li F, et al. Quantitative evaluation of liver function with use of gadoxetate disodium-enhanced MR imaging. Radiology 2011;260:727-733.

25. Nilsson H, Nordell A, Vargas R, Douglas L, Jonas E, Blomqvist L. Assessment of hepatic extraction fraction and input relative blood flow using dynamic hepatocyte-specific contrast-enhanced MRI. J Magn Reson Imaging 2009;29:1323-1331.

26. Nilsson H, Blomqvist L, Douglas L, Nordell A, Jonas E. Assessment of liver function in primary biliary cirrhosis using GdEOB-DTPA-enhanced liver MRI. HPB (Oxford) 2010;12:567-576. 
27. Sourbron S, Sommer WH, Reiser MF, Zech CJ. Combined quantification of liver perfusion and function with dynamic gadoxetic acid-enhanced MR imaging. Radiology 2012;263:874-883.

28. van den Broek MA, van Dam RM, Malago M, Dejong $\mathrm{CH}$, van Breukelen GJ, Olde Damink SW. Feasibility of randomized controlled trials in liver surgery using surgery-related mortality or morbidity as endpoint. Br J Surg 2009;96:1005-1014.

29. Wright M, Tidy C.Liver tumours. Patient.co.uk. Available at: http://www.patient.co.uk/doctor/Liver-Tumours.htm. Accessed October 16, 2012.

30. Semelka RC, Helmberger TK. Contrast agents for MR imaging of the liver. Radiology 2001;218:27-38.

31. Nino-Murcia M, Olcott EW, Jeffrey RB Jr, Lamm RL, Beaulieu CF, Jain KA. Focal liver lesions: pattern-based classification scheme for enhancement at arterial phase CT. Radiology 2000; 215:746-751.

32. Semelka RC, Hussain SM, Marcos HB, Woosley JT. Perilesional enhancement of hepatic metastases: correlation between MR imaging and histopathologic findings-initial observations. Radiology 2000;215:89-94.

33. Scurr ED, Collins DJ, Temple L, Karanjia N, Leach MO, Koh DM. Appearances of colorectal hepatic metastases at diffusionweighted MRI compared with histopathology: initial observations. Br J Radiol 2012;85:225-230.

34. Kele PG, van der Jagt EJ. Diffusion weighted imaging in the liver. World J Gastroenterol 2010;16:1567-1576.

35. Danet IM, Semelka RC, Leonardou P, et al. Spectrum of MRI appearances of untreated metastases of the liver. AJR Am J Roentgenol 2003;181:809-817.

36. Niekel MC, Bipat S, Stoker J. Diagnostic imaging of colorectal liver metastases with CT, MR imaging, FDG PET, and/or FDG PET/CT: a meta-analysis of prospective studies including patients who have not previously undergone treatment. Radiology 2010;257:674-684.

37. Jeong HT, Kim MJ, Park MS, et al. Detection of liver metastases using gadoxetic-enhanced dynamic and 10- and 20-minute delayed phase MR imaging. J Magn Reson Imaging 2012;35: 635-643.

38. Ichikawa T, Saito K, Yoshioka N, et al. Detection and characterization of focal liver lesions: a Japanese phase III, multicenter comparison between gadoxetic acid disodium-enhanced magnetic resonance imaging and contrast-enhanced computed tomography predominantly in patients with hepatocellular carcinoma and chronic liver disease. Invest Radiol 2010;45:133-141.

39. Raman SS, Leary C, Bluemke DA, et al. Improved characterization of focal liver lesions with liver-specific gadoxetic acid disodium-enhanced magnetic resonance imaging: a multicenter phase 3 clinical trial. J Comput Assist Tomogr 2010;34:163-172.

40. Kim YK, Park G, Kim CS, Yu HC, Han YM. Diagnostic efficacy of gadoxetic acid-enhanced MRI for the detection and characterisation of liver metastases: comparison with multidetector-row CT. Br J Radiol 2012;85:539-547.

41. Kim T, Murakami T, Takahashi S, Hori M, Tsuda K, Nakamura H. Diffusion-weighted single-shot echoplanar MR imaging for liver disease. AJR Am J Roentgenol 1999;173:393-398.

42. Koh DM, Collins DJ, Wallace T, Chau I, Riddell AM. Combining diffusion-weighted MRI with Gd-EOB-DTPA-enhanced MRI improves the detection of colorectal liver metastases. Br J Radiol 2012;85:980-989.

43. Nagle SK, Busse RF, Brau AC, et al. High resolution navigated three-dimensional T(1) -weighted hepatobiliary MRI using gadoxetic acid optimized for 1.5 tesla. J Magn Reson Imaging 2012; 36:890-899.

44. Bashir MR, Husarik DB, Ziemlewicz TJ, Gupta RT, Boll DT, Merkle EM. Liver MRI in the hepatocyte phase with gadoliniumEOB-DTPA: does increasing the flip angle improve conspicuity and detection rate of hypointense lesions? J Magn Reson Imaging 2012;35:611-616.

45. Ahn SS, Kim MJ, Lim JS, Hong HS, Chung YE, Choi JY. Added value of gadoxetic acid-enhanced hepatobiliary phase MR imaging in the diagnosis of hepatocellular carcinoma. Radiology 2010;255:459-466.

46. Di Martino M, Marin D, Guerrisi A, et al. Intraindividual comparison of gadoxetate disodium-enhanced MR imaging and 64section multidetector CT in the Detection of hepatocellular carcinoma in patients with cirrhosis. Radiology 2010;256:806-816.
47. Golfieri R, Renzulli M, Lucidi V, Corcioni B, Trevisani F, Bolondi L. Contribution of the hepatobiliary phase of Gd-EOBDTPA-enhanced MRI to Dynamic MRI in the detection of hypovascular small $(\leq 2 \mathrm{~cm}) \mathrm{HCC}$ in cirrhosis. Eur Radiol 2011;21: 1233-1242.

48. Haradome H, Grazioli L, Tinti R, et al. Additional value of gadoxetic acid-DTPA-enhanced hepatobiliary phase MR imaging in the diagnosis of early-stage hepatocellular carcinoma: comparison with dynamic triple-phase multidetector CT imaging. J Magn Reson Imaging 2011;34:69-78.

49. Kim SH, Kim SH, Lee J, et al. Gadoxetic acid-enhanced MRI versus triple-phase MDCT for the preoperative detection of hepatocellular carcinoma. AJR Am J Roentgenol 2009;192:1675-1681.

50. Sun HY, Lee JM, Shin CI, et al. Gadoxetic acid-enhanced magnetic resonance imaging for differentiating small hepatocellular carcinomas ( $\leq 2 \mathrm{~cm}$ in diameter) from arterial enhancing pseudolesions: special emphasis on hepatobiliary phase imaging. Invest Radiol 2010;45:96-103.

51. Motosugi U, Ichikawa T, Sou H, et al. Distinguishing hypervascular pseudolesions of the liver from hypervascular hepatocellular carcinomas with gadoxetic acid-enhanced MR imaging. Radiology 2010;256:151-158.

52. Kobayashi S, Matsui O, Gabata T, et al. Gadolinium ethoxybenzyl diethylenetriamine pentaacetic Acid-enhanced magnetic resonance imaging findings of borderline lesions at high risk for progression to hypervascular classic hepatocellular carcinoma. J Comput Assist Tomogr 2011;35:181-186.

53. Kumada T, Toyoda H, Tada $\mathrm{T}$, et al. Evolution of hypointense hepatocellular nodules observed only in the hepatobiliary phase of gadoxetate disodium-enhanced MRI. AJR Am J Roentgenol 2011;197:58-63.

54. Filippone A, Blakeborough A, Breuer J, et al. Enhancement of liver parenchyma after injection of hepatocyte-specific MRI contrast media: a comparison of gadoxetic acid and gadobenate dimeglumine. J Magn Reson Imaging 2010;31:356-364.

55. Motosugi U, Ichikawa T, Sano K, et al. Double-dose gadoxetic Acid-enhanced magnetic resonance imaging in patients with chronic liver disease. Invest Radiol 2011;46:141-145.

56. Higaki A, Tamada $\mathrm{T}$, Sone $\mathrm{T}$, et al. Potential clinical factors affecting hepatobiliary enhancement at Gd-EOB-DTPA-enhanced MR imaging. Magn Reson Imaging 2012;30:689-693.

57. Kanki A, Tamada T, Higaki A, et al. Hepatic parenchymal enhancement at Gd-EOB-DTPA-enhanced MR imaging: correlation with morphological grading of severity in cirrhosis and chronic hepatitis. Magn Reson Imaging 2012;30:356-360.

58. Kubota K, Tamura T, Aoyama N, et al. Correlation of liver parenchymal gadolinium-ethoxybenzyl diethylenetriaminepentaacetic acid enhancement and liver function in humans with hepatocellular carcinoma. Oncol Lett 2012;3:990-994.

59. Nakamura S, Awai K, Utsunomiya D, et al. Chronological evaluation of liver enhancement in patients with chronic liver disease at Gd-EOB-DTPA-enhanced 3-T MR imaging: does liver function correlate with enhancement? Jpn J Radiol 2012;30:25-33.

60. Motosugi U, Ichikawa T, Muhi A, et al. Magnetic resonance elastography as a predictor of insufficient liver enhancement on gadoxetic Acid-enhanced hepatocyte-phase magnetic resonance imaging in patients with type $\mathrm{C}$ hepatitis and child-pugh class a disease. Invest Radiol 2012;47:566-570.

61. Motosugi U, Ichikawa $\mathrm{T}$, Sou $\mathrm{H}$, et al. Liver parenchymal enhancement of hepatocyte-phase images in Gd-EOB-DTPAenhanced MR imaging: which biological markers of the liver function affect the enhancement? J Magn Reson Imaging 2009; 30:1042-1046.

62. Tajima $\mathrm{T}$, Takao $\mathrm{H}$, Akai $\mathrm{H}$, et al. Relationship between liver function and liver signal intensity in hepatobiliary phase of gadolinium ethoxybenzyl diethylenetriamine pentaacetic acidenhanced magnetic resonance imaging. J Comput Assist Tomogr 2010;34:362-366.

63. Gschwend S, Ebert W, Schultze-Mosgau M, Breuer J. Pharmacokinetics and imaging properties of Gd-EOB-DTPA in patients with hepatic and renal impairment. Invest Radiol 2011;46:556-566.

64. Kim HY, Choi JY, Park CH, et al. Clinical factors predictive of insufficient liver enhancement on the hepatocyte-phase of GdEOB-DTPA-enhanced magnetic resonance imaging in patients with liver cirrhosis. J Gastroenterol [Epub ahead of print]. 
65. Tschirch FT, Struwe A, Petrowsky H, Kakales I, Marincek B, Weishaupt D. Contrast-enhanced MR cholangiography with GdEOB-DTPA in patients with liver cirrhosis: visualization of the biliary ducts in comparison with patients with normal liver parenchyma. Eur Radiol 2008;18:1577-1586.

66. Tsuboyama T, Onishi H, Kim T, et al. Hepatocellular carcinoma: hepatocyte-selective enhancement at gadoxetic acid-enhanced MR imaging--correlation with expression of sinusoidal and canalicular transporters and bile accumulation. Radiology 2010;255: 824-833.

67. Tsuda N, Matsui O. Cirrhotic rat liver: reference to transporter activity and morphologic changes in bile canaliculi--gadoxetic acid-enhanced MR imaging. Radiology 2010;256:767-773.

68. Kim MJ, Rhee HJ, Jeong HT. Hyperintense lesions on gadoxetate disodium-enhanced hepatobiliary phase imaging. AJR Am J Roentgenol 2012;199:W575-W586.

69. Kobayashi S, Matsui O, Gabata T, et al. Relationship between signal intensity on hepatobiliary phase of gadolinium ethoxybenzyl diethylenetriaminepentaacetic acid (Gd-EOB-DTPA) enhanced MR imaging and prognosis of borderline lesions of hepatocellular carcinoma. Eur J Radiol 2012;81:3002-3009.

70. Sano K, Motosugi U, Ichikawa T, Morisaka H, Ichikawa S, Araki T.The outcome of hypovascular nodules showing hyperintense on hepatocyte-phase of gadoxetic acid-enhanced magnetic resonance imaging: when does it become a conventional HCC? 4 years follow-up study. In: Proceedings of the Annual Meeting of Radiological Society of North America, Chicago, November 2530, 2012.

71. Bartolozzi C, Battaglia V, Bargellini I, et al. Contrast-enhanced magnetic resonance imaging of 102 nodules in cirrhosis: correlation with histological findings on explanted livers. Abdominal Imaging 2013;38:290-296.

72. Nakashima O, Sugihara S, Kage M, Kojiro M. Pathomorphologic characteristics of small hepatocellular carcinoma: a special reference to small hepatocellular carcinoma with indistinct margins. Hepatology 1995;22:101-105.

73. Takayama T, Makuuchi M, Hirohashi S, et al. Early hepatocellular carcinoma as an entity with a high rate of surgical cure Hepatology 1998;28:1241-1246.

74. Kogita S, Imai Y, Okada M, et al. Gd-EOB-DTPA-enhanced magnetic resonance images of hepatocellular carcinoma: correlation with histological grading and portal blood flow. Eur Radiol 2010; 20:2405-2413

75. Sugitani S, Sakamoto M, Ichida T, Genda T, Asakura H, Hirohashi S. Hyperplastic foci reflect the risk of multicentric development of human hepatocellular carcinoma. J Hepatol 1998;28:1045-1053.

76. Motosugi U, Ichikawa T, Sano K, et al. Outcome of hypovascular hepatic nodules revealing no gadoxetic acid uptake in patients with chronic liver disease. J Magn Reson Imaging 201 1;34:88-94.

77. Akai H, Matsuda I, Kiryu S, et al. Fate of hypointense lesions on Gd-EOB-DTPA-enhanced magnetic resonance imaging. Eur J Radiol 2012;81:2973-2977.

78. Takayama Y, Nishie A, Nakayama T, et al. Hypovascular hepatic nodule showing hypointensity in the hepatobiliary phase of gadoxetic acid-enhanced MRI in patients with chronic liver disease: prediction of malignant transformation. Eur $\mathrm{J}$ Radiol 2012;81:3072-3078.

79. Bruix J, Sherman M. Management of hepatocellular carcinoma: an update. Hepatology 2011;53:1020-1022.

80. Park G, Kim YK, Kim CS, Yu HC, Hwang SB. Diagnostic efficacy of gadoxetic acid-enhanced MRI in the detection of hepatocellular carcinomas: comparison with gadopentetate dimeglumine. $\mathrm{Br}$ J Radiol 2010;83:1010-1016.

81. Akai H, Kiryu S, Matsuda I, et al. Detection of hepatocellular carcinoma by Gd-EOB-DTPA-enhanced liver MRI: comparison with triple phase 64 detector row helical CT. Eur J Radiol 2011 80:310-315.

82. Inoue $\mathrm{T}$, Kudo $\mathrm{M}$, Komuta $\mathrm{M}$, et al Assessment of Gd-EOBDTPA-enhanced MRI for HCC and dysplastic nodules and comparison of detection sensitivity versus MDCT. J Gastroenterol 2012;47:1036-1047.

83. Leoni S, Piscaglia F, Golfieri R, et al. The impact of vascular and nonvascular findings on the noninvasive diagnosis of small hepatocellular carcinoma based on the EASL and AASLD criteria. Am J Gastroenterol 2010;105:599-609.
84. Bolondi L, Gaiani S, Celli N, et al. Characterization of small nodules in cirrhosis by assessment of vascularity: the problem of hypovascular hepatocellular carcinoma. Hepatology 2005;42:27-34.

85. Forner A, Llovet JM, Bruix J. Hepatocellular carcinoma. Lancet 2012;379:1245-1255.

86. Mazzaferro V, Llovet JM, Miceli R, et al. Predicting survival after liver transplantation in patients with hepatocellular carcinoma beyond the Milan criteria: a retrospective, exploratory analysis. Lancet Oncol 2009;10:35-43.

87. Lencioni R, Llovet JM. Modified RECIST (mRECIST) assessment for hepatocellular carcinoma. Semin Liver Dis 2010;30:52-60.

88. Hwang J, Kim SH, Kim YS, et al. Gadoxetic acid-enhanced MRI versus multiphase multidetector row computed tomography for evaluating the viable tumor of hepatocellular carcinomas treated with image-guided tumor therapy. J Magn Reson Imaging 2010; 32:629-638.

89. Chong YS, Kim YK, Lee MW, et al. Differentiating mass-forming intrahepatic cholangiocarcinoma from atypical hepatocellular carcinoma using gadoxetic acid-enhanced MRI. Clin Radiol 2012;67:766-773.

90. Kim $\mathrm{SH}$, Lee $\mathrm{CH}$, Kim $\mathrm{BH}$, et al. Typical and atypical imaging findings of intrahepatic cholangiocarcinoma using gadolinium ethoxybenzyl diethylenetriamine pentaacetic Acid-enhanced magnetic resonance imaging. J Comput Assist Tomogr 2012;36: 704-709.

91. Kim YK, Kim CS, Han YM, Yu HC, Choi D. Detection of small hepatocellular carcinoma: intraindividual comparison of gadoxetic acid-enhanced MRI at 3.0 and 1.5 T. Invest Radiol 2011;46: 383-389.

92. Baek CK, Choi JY, Kim KA, et al. Hepatocellular carcinoma in patients with chronic liver disease: a comparison of gadoxetic acid-enhanced MRI and multiphasic MDCT. Clin Radiol 2012; 67:148-156.

93. Chou CT, Chen YL, Su WW, Wu HK, Chen RC. Characterization of cirrhotic nodules with gadoxetic acid-enhanced magnetic resonance imaging: the efficacy of hepatocyte-phase imaging. J Magn Reson Imaging 2010;32:895-902.

94. Doo KW, Lee CH, Choi JW, Lee J, Kim KA, Park CM. "Pseudo washout" sign in high-flow hepatic hemangioma on gadoxetic acid contrast-enhanced MRI mimicking hypervascular tumor. AJR Am J Roentgenol 2009;193:W490-W496.

95. Frericks BB, Loddenkemper C, Huppertz A, et al. Qualitative and quantitative evaluation of hepatocellular carcinoma and cirrhotic liver enhancement using Gd-EOB-DTPA. AJR Am J Roentgenol 2009;193:1053-1060.

96. Golfieri R, Grazioli L, Orlando E, et al. Which is the best MRI marker of malignancy for atypical cirrhotic nodules: hypointensity in hepatobiliary phase alone or combined with other features? Classification after Gd-EOB-DTPA administration. J Magn Reson Imaging 2012;36:648-657

97. Goshima S, Kanematsu M, Watanabe H, et al. Gadoxetate disodium-enhanced MR imaging: differentiation between earlyenhancing non-tumorous lesions and hypervascular hepatocellular carcinomas. Eur J Radiol 2011;79:e108-e112.

98. Hwang J, Kim SH, Lee MW, Lee JY. Small $(<2 \mathrm{~cm})$ hepatocellular carcinoma in patients with chronic liver disease: comparison of gadoxetic acid-enhanced $3.0 \mathrm{~T}$ MRI and multiphasic 64multirow detector CT. Br J Radiol 2012;85:e314-e322.

99. Jung G, Breuer J, Poll LW, et al. Imaging characteristics of hepatocellular carcinoma using the hepatobiliary contrast agent Gd-EOB-DTPA. Acta Radiol 2006;47:15-23.

100. Kim YK, Kim CS, Han YM, Park G. Detection of small hepatocellular carcinoma: can gadoxetic acid-enhanced magnetic resonance imaging replace combining gadopentetate dimeglumineenhanced and superparamagnetic iron oxide-enhanced magnetic resonance imaging? Invest Radiol 2010;45:740-746.

101. Kim YK, Kim CS, Han YM, Park G, Hwang SB, Yu HC. Comparison of gadoxetic acid-enhanced MRI and superparamagnetic iron oxide-enhanced MRI for the detection of hepatocellular carcinoma. Clin Radiol 2010;65:358-365.

102. Lee S, Kim SH, Park CK, Kim YS, Lee WJ, Lim HK. Comparison between areas with Gd-EOB-DTPA uptake and without in hepatocellular carcinomas on Gd-EOB-DTPA-enhanced hepatobiliary-phase MR imaging: pathological correlation. J Magn Reson Imaging 2010;32:719-725. 
103. Marin D, Di Martino M, Guerrisi A, et al. Hepatocellular carcinoma in patients with cirrhosis: qualitative comparison of gadobenate dimeglumine-enhanced MR imaging and multiphasic 64-section CT. Radiology 2009;251:85-95.

104. Mita K, Kim SR, Kudo M, et al. Diagnostic sensitivity of imaging modalities for hepatocellular carcinoma smaller than $2 \mathrm{~cm}$. World J Gastroenterol 2010;16:4187-4192.

105. Onishi H, Kim T, Imai Y, et al. Hypervascular hepatocellular carcinomas: detection with gadoxetate disodium-enhanced MR imaging and multiphasic multidetector CT. Eur Radiol 2012;22:845-854.

106. Park Y, Kim SH, Kim SH, et al. Gadoxetic acid (Gd-EOB-DTPA)enhanced MRI versus gadobenate dimeglumine (Gd-BOPTA) enhanced MRI for preoperatively detecting hepatocellular carcinoma: an initial experience. Korean J Radiol 2010;11:433-440.
107. Rhee H, Kim MJ, Park YN, Choi JS, Kim KS. Gadoxetic acidenhanced MRI findings of early hepatocellular carcinoma as defined by new histologic criteria. J Magn Reson Imaging 2012; 35:393-398.

108. Sano K, Ichikawa T, Motosugi U, et al. Imaging study of early hepatocellular carcinoma: usefulness of gadoxetic acidenhanced MR imaging. Radiology 2011;261:834-844.

109. Shinagawa Y, Sakamoto K, Fujimitsu R, et al. Pseudolesion of the liver observed on gadoxetate disodium-enhanced magnetic resonance imaging obtained shortly after transarterial chemoembolization for hepatocellular carcinoma. Jpn J Radiol 2010;28:483-488.

110. Suh YJ, Kim MJ, Choi JY, Park YN, Park MS, Kim KW. Differentiation of hepatic hyperintense lesions seen on gadoxetic acidenhanced hepatobiliary phase MRI. AJR Am J Roentgenol 2011; 197:W44-W52. 